\title{
Magnitud y variación de la mastitis clínica y sus costos asociados en rodeos lecheros de Argentina
}

\author{
Magnitude and variation of clinical mastitis and its associated costs \\ in dairy herds of Argentina

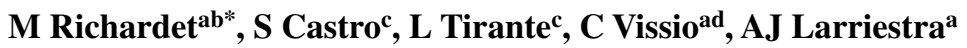

\begin{abstract}
A survey was conducted to evaluate the magnitude and variation of clinical mastitis among dairy herds. Prevalence, cumulative incidence and incidence rate were estimated, exploring risk factors at cow level and economic consequences. A cross sectional convenience sample of 2,535 Holstein-Friesian milking cows from 19 herds was the study population. Herd prevalence was estimated the day of the visit. Cows that showed no clinical signs were followed during 15 days to get incidence estimates. Mixed logistic models and survival analysis were applied. Using prevalence data, each case was monetized considering milk discharged, treatment costs and estimated milk losses until dry-off. Overall gross prevalence was 2.5 cases $/ 100$ cows $(95 \% \mathrm{CI}=1.9-3.1)$, cumulative incidence was 3.9 new cases $/ 100$ milking cows $(95 \% \mathrm{CI}=3.1-4.7)$ and incidence rate was 2.7 new cases $/ 1.000$ cow-days $(95 \% \mathrm{CI}=2.2-3.3)$. The herds medians were $1.69(\mathrm{q} 1=0, \mathrm{q} 3=4.2), 3.6(\mathrm{q} 1=2.0, \mathrm{q} 3=4.6)$ and $0.25(\mathrm{q} 1=0.14, \mathrm{q} 3=0.42)$, respectively. Cows during early lactation showed higher odds of being a prevalent case $(\mathrm{OR}=1.69 ; 95 \% \mathrm{CI}=1.01-2.85)$. In cows, cumulative incidence $(\mathrm{OR}=3.36$; $95 \% \mathrm{CI}=1.84-6.11)$ and incidence rate $(\mathrm{HR}=3.83 ; 95 \% \mathrm{CI}=2.05-7.15)$ were three times higher compared with heifers, after the adjustment for days in milk and herd. Average cost for case was US\$ 185.01 and US\$ 143.03 in cows and heifers, respectively, and the average losses reached up to US\$ $4.50 /$ milking cow $(95 \% \mathrm{CI}=3.38-5.61)$. This is the first study documenting the clinical mastitis frequency and losses in dairy herds of Argentina.

Key words: clinical mastitis, prevalence, incidence, economic losses.
\end{abstract}

\begin{abstract}
RESUMEN. Se llevó a cabo un estudio para evaluar la magnitud y variación de la mastitis clínica en predios lecheros. Se estimaron la prevalencia, incidencia acumulada y tasa de incidencia, explorando factores de riesgo a nivel de vaca y las consecuencias económicas de los casos clínicos. Una muestra de conveniencia de 2.535 vacas Holstein-Friesian en lactación de 19 rodeos lecheros constituyó la población en estudio. En cada predio, la prevalencia fue estimada el día de la visita. Las vacas sin mastitis fueron monitoreadas 15 días para estimar la incidencia. Se aplicaron modelos logísticos y de supervivencia mixtos. Los casos prevalentes fueron valuados considerando la leche descartada, tratamiento y disminución de la producción en el resto de la lactancia. La prevalencia cruda fue de 2,5 casos/100 vacas (IC 95\% = 1,9-3,1), la incidencia acumulada de 3,9 casos nuevos/100 vacas (IC 95\% = 3,1-4,7) y la tasa de incidencia de 2,7 casos nuevos/1.000 vacas-días a riesgo (IC 95\% = 2,2-3,3). La mediana de rodeo fue de 1,69 $(\mathrm{q} 1=0 ; \mathrm{q} 3=4,2)$, $3,6(\mathrm{q} 1=2,0 ; \mathrm{q} 3=4,6)$ y $0,25(\mathrm{q} 1=0,14 ; \mathrm{q} 3=0,42)$, respectivamente. Los casos fueron más frecuentes en lactancia temprana $(\mathrm{OR}=1,69 ; \mathrm{IC} 95 \%=1,01-2,85)$. En vacas, tanto la incidencia acumulada $(\mathrm{OR}=3,36 ; \mathrm{IC} 95 \%=1,84-6,11)$ como la tasa de incidencia $(\mathrm{HR}=3,83$; IC $95 \%=2,05-7,15)$ fueron tres veces mayores. El costo de un caso fue de US\$185,01 y US\$143,03 para vacas y vaquillonas, respectivamente. La pérdida promedio fue de 4,50 US\$/vaca en ordeña (IC 95\% = 3,38-5,61). Este es el primer estudio que aborda la distribución e impacto de la mastitis clínica en rodeos lecheros en Argentina.

Palabras clave: mastitis clínica, prevalencia, incidencia, pérdidas económicas.
\end{abstract}

\section{INTRODUCCIÓN}

La mastitis es la enfermedad más costosa del ganado lechero a nivel mundial (Hogeveen y col 2011). Las pérdidas directas debidas a mastitis se deben principalmente

Aceptado: 26.11.2015.

aFacultad de Agronomía y Veterinaria, Universidad Nacional de Río Cuarto, Río Cuarto, Argentina.

bBecaria de CONICET, Argentina.

${ }^{\mathrm{c}}$ Asociación pro Calidad de la Leche y sus Derivados-APROCAL, Buenos Aires, Argentina.

${ }^{\mathrm{d}}$ CIC CONICET, Argentina.

\# Este trabajo fue financiado por SECyT (Secretaría de Ciencia y Técnica, Universidad Nacional de Río Cuarto) y APROCAL (Asociación pro Calidad de la Leche y sus Derivados, CABA, Argentina).

*Autor de correspondencia: M Richardet; Ruta Nac. 36, Km. 601, Código Postal X5804BYA Río Cuarto, Córdoba, Argentina; mrichardet@hotmail.com a la disminución de la producción de leche asociada a las formas clínica y subclínica de la enfermedad (Wilson y col 2004, Vissio y col 2015). Otras pérdidas son atribuidas al descarte de leche anormal o con presencia de antibióticos, gastos en medicación, pago de honorarios veterinarios, fallas reproductivas, descarte de vacas y costos de reemplazo de animales (Hogeveen y col 2011).

Numerosos factores, como las características individuales de las vacas (Olde Riekerink y col 2007), los componentes ambientales y de manejo (Nielsen 2009) y el rodeo de pertenencia (Barkema y col 1999, Nyman y col 2007), pueden estar asociados a la prevalencia e incidencia de la mastitis.

La mastitis es una enfermedad que evoluciona con el sistema productivo y de acuerdo con las condiciones de control imperantes en el predio (Bradley 2002). Según estas circunstancias, la importancia relativa de las formas 
clínica y subclínica de esta enfermedad y la de los agentes etiológicos involucrados pueden variar (Zadoks y Fitzpatrick 2009). Es así que en rodeos que tienen un plan de salud mamaria establecido y basado en prácticas de reconocida eficacia, según avanza el control, la fisonomía de la mastitis se va modificando (Bradley 2002). En este sentido, el cambio en los sistemas de producción hacia un modelo con mayor intensificación y la consolidación del plan de salud mamaria conocido como "Plan de los cinco puntos" (desinfección de pezones posordeño, terapia de vaca seca, tratamiento apropiado de casos clínicos, descarte de vacas con infección crónica, mantenimiento periódico de la máquina de ordeñar), contribuyen a la emergencia de nuevos patrones de la enfermedad con una mayor frecuencia de mastitis clínica (MC) y un predominio de infecciones mamarias producidas por agentes ambientales (Zadoks y Fitzpatrick 2009).

La falta de estudios previos que aborden esta temática en nuestro país motivó a la realización del presente trabajo, cuyo objetivo fue realizar una primera aproximación al estudio de la variación de la prevalencia e incidencia de la MC y de sus consecuencias económicas en rodeos lecheros con buenos estándares de calidad de leche en tanque y que aplican regularmente un plan de salud mamaria convencional.

\section{MATERIAL Y MÉTODOS}

\section{ESTABLECIMIENTOS LECHEROS ESTUDIADOS}

El total de establecimientos estudiados estuvo constituido por 19 predios que consintieron su participación mediante una invitación realizada por la Asociación Pro Calidad de Leche de Argentina (AProCal) por medio del veterinario asesor. Debido al carácter colaborativo que tuvo este trabajo y a la necesidad de obtener resultados en el corto plazo, dicho estudio se desarrolló en un tiempo corto, a fin de evaluar de manera rápida una gran cantidad de datos obtenidos de los predios involucrados. Los participantes provenían de las provincias de Buenos Aires y Santa Fe, y fueron visitados entre febrero y mayo del 2011. El período de recolección de datos en cada establecimiento fue de 15 días. La población total de vacas en ordeño (VO) fue de 6.240, en su mayoría de raza Holando Argentino (Holstein-Friesian; 94,7\%), con un promedio de $337 \mathrm{VO}$ por predio. El sistema pastoril o semipastoril fue el predominante $(79 \%)$, mientras que el $21 \%$ de los establecimientos había adoptado el encierre en corrales abiertos.

En los predios estudiados se realizaba ordeño mecánico $(\geq 2)$, con una rutina que incluía el registro de los casos de MC, el tratamiento antibiótico intramamario al secado y la aplicación de antiséptico de pezones posordeño. Además, en el $47 \%$ de los predios se realizaba la desinfección de los pezones previa al ordeño. Los predios participantes reportaron recuentos en tanque menores a 300.000 células somáticas $/ \mathrm{mL}$ y menores a $20.000 \mathrm{ufc} / \mathrm{mL}$ en los tres meses previos a la realización del estudio.

\section{SELECCIÓN DE LOS ANIMALES EN CADA PREDIO}

El tamaño de muestra en cada predio fue estimado por la fórmula $\left[\mathrm{Z}^{2} * \mathrm{P} *(1-\mathrm{P}) / \mathrm{d}^{2}\right]$ (Thrusfield 2007), con una confianza del $95 \%(\mathrm{Z}=1,96)$, asumiendo una prevalencia de $\mathrm{MC}(\mathrm{P})$ del $4 \%$ y una precisión (d) del $2 \%(2-6 \%)$, realizando el ajuste correspondiente según el número total de $\mathrm{VO}$ el día de la visita. Las vacas fueron seleccionadas aleatoriamente mediante un muestreo sistemático implementado en la sala de ordeño y siguiendo la pauta de trabajo sugerida por Vissio (2012). Un total de 2.535 VO fueron seleccionadas y examinadas, la fracción de muestreo promedio fue de $40,6 \%$, con un rango por predio de $25,2 \%-65,9 \%$.

\section{DEFINICIÓN DEL CASO DE MASTITIS}

Se entendió como caso a toda vaca cuya leche presentara alteraciones (cambios en color, textura, consistencia, etc.) al momento del despunte, asociadas o no a signos clínicos locales (a nivel de la ubre) o sistémicos. Cualquier episodio clínico ocurrido dentro de las dos semanas posteriores al evento de MC fue considerado como el mismo episodio (Halasa y col 2009). La detección de casos fue llevada a cabo por profesionales capacitados, estandarizándose la forma de trabajo mediante un entrenamiento conjunto de todos los veterinarios voluntarios. De cada animal muestreado se registró su número de identificación (RP), número ordinal de parto y días en leche (DEL). Los datos relevados fueron registrados en planillas elaboradas para tal fin, donde se asentó la fecha de presentación del caso de MC y la fecha de secado, descarte o muerte si correspondiese. El período de seguimiento de los animales en cada predio fue de 15 días a partir de la primera visita.

\section{FRECUENCIA DE MASTITIS CLÍNICA}

A partir del conjunto de vacas seleccionadas y examinadas el primer día se calculó la prevalencia puntual (Thrusfield 2007), definida como el cociente entre el número de vacas que presentaron $\mathrm{MC}$ y el número total de vacas seleccionadas ese día. Dicho indicador fue expresado por cada $100 \mathrm{VO}$ en el día de la visita y para el predio de referencia. Posteriormente todas aquellas vacas seleccionadas que no poseían MC fueron monitoreadas durante los siguientes 15 días, a los efectos de identificar los casos incidentes. Solo se registró el primer caso incidente durante el período de observación, y las recurrencias de $\mathrm{MC}$ no fueron consideradas como nuevos episodios.

Durante este lapso se registró, además, la fecha de secado, descarte o muerte para cada vaca. A partir de estos datos se calculó la incidencia, tanto en la forma de 
proporción, incidencia acumulada (IA), como en la de tasa de incidencia (TI) (Thrusfield 2007). Las fórmulas empleadas fueron: a) $I A=$ Número total de vacas que presentaron casos incidentes de MC durante los 15 días de observación/ Número total de vacas muestreadas sanas al inicio del período de observación; b) TI = Número total de vacas que presentaron episodios incidentes de MC/Vaca-días a riesgo. El denominador en el inciso b) se dedujo sumando los días que cada vaca estuvo en monitoreo, lo que implicó el descuento del número de días desde la aparición del caso hasta finalizar el período de observación, así como también los días en que la vaca no estuvo en ordeño por diferentes motivos (secado, descarte o muerte).

\section{ANÁLISIS ESTADÍSTICO}

Se analizaron la prevalencia, IA y TI de MC para el conjunto de las vacas estudiadas y por predio (medidas de frecuencias crudas). Las medidas de frecuencia específicas fueron analizadas considerando dos factores individuales de la vaca, la paridad del animal (vaquillona: 1 parto; vaca: 2 o más partos), y los DEL ( $\leq 90$ días y $>90$ días). Debido a que se trabajó con una muestra aleatoria de vacas, se calcularon los intervalos de confianza para cada una de las medidas de frecuencia (Thursfield 2007).

\section{ANÁLISIS DE FACTORES DE RIESGO CONSIDERANDO EL EFECTO CONTEXTUAL DEL PREDIO LECHERO}

Los factores de riesgo analizados fueron paridad y DEL. Para esto se ajustaron tres modelos, dos de regresión logística (Le 1997) para evaluar los factores asociados a la probabilidad de ser un caso prevalente o un caso incidente, y un modelo de regresión proporcional de Cox (Le 1997) para evaluar los factores asociados a la TI. Las interacciones entre paridad y DEL fueron analizadas en los tres modelos y, además, para contabilizar la variación en el riesgo de MC debido al contexto de manejo del rodeo, el predio fue incluido como efecto aleatorio en todos ellos. En estos tipos de modelos se puede calcular la proporción de la variación atribuible al efecto contextual (predio), utilizando el coeficiente de correlación intraclase (CCI, ९) en la forma descrita por Snijders y Bosker (2011);

$$
\rho=\sigma_{t}^{2} /\left(\sigma_{t}^{2}+\pi^{2} / 3\right)
$$

donde $\sigma^{2}$ es la varianza atribuible al predio.

Para evaluar el ajuste de los modelos se realizó el análisis gráfico de los residuos. Todos los análisis fueron llevados a cabo en el programa STATA versión 11.

\section{IMPACTO ECONÓMICO DE LA MASTITIS CLÍNICA}

Las pérdidas monetarias derivadas del episodio de MC en este estudio fueron estimadas considerando los costos de la leche descartada y del tratamiento (Wolfová y col 2006). Se asumió, según los productos disponibles en el mercado utilizados en los predios, que cada vaca fue tratada en promedio con tres aplicaciones de antibiótico intramamario a un precio de US\$ 3 cada una (cotización del 30/08/2013), y que la leche fue descartada durante cinco días. El precio de la leche en dólares fue de 0,37 US\$/litro (cotización del 30/08/2013). La producción diaria promedio de leche fue de $30,83 \mathrm{~L} / \mathrm{d}$ para vacas y de 25,00 L/d para vaquillonas.

Además, considerando los DEL al momento del evento y asumiendo una duración de la lactancia promedio de 10 meses, se dedujeron las pérdidas productivas posteriores al episodio y atribuibles exclusivamente al mismo, discriminadas entre vacas y vaquillonas, utilizando las estimaciones realizadas por Tirante y col (2013). Posteriormente se obtuvo una valoración final constituida por los costos del episodio de MC registrado más las pérdidas estimadas para el resto de la lactancia debidas a este caso. El costo monetario de las pérdidas se expresó en dólares.

\section{RESULTADOS}

\section{MEDIDAS DE FRECUENCIA CRUDAS Y ESPECÍFICAS}

Sobre 2.535 VO muestreadas en este estudio, la prevalencia cruda de MC fue de 2,53 (IC 95\% = 1,89-3,16) casos por cada $100 \mathrm{VO}$. Basados en los casos incidentes detectados durante los quince días posteriores a la primera visita, se determinó una IA cruda de 3,93 (IC 95\% = 3,144,71 ) casos nuevos por cada $100 \mathrm{VO}$ y una TI cruda de 2,70 (IC 95\% = 2,20-3,30) casos nuevos por cada 1.000 VO-días a riesgo.

Al examinar las medidas de frecuencia específicas utilizando los datos de estas 2.535 VO se apreció que la prevalencia en vacas fue casi dos veces mayor que la evidenciada en vaquillonas (cuadro 1). En cuanto a los DEL, la presentación de MC durante los primeros 90 días fue también el doble respecto de lactancias más tardías (cuadro 1). La evaluación de la IA específica mostró que las vacas presentaron un riesgo de MC substancialmente mayor que las vaquillonas, siendo de menor magnitud en el caso de los DEL (cuadro 1).

A nivel de predio $(n=19)$, la mediana de la prevalencia, IA y TI de MC fue de 1,69\% (percentil 25\% = 0,00; percentil $75 \%=4,20), 3,60 \%$ (percentil 25\% = 2,00; percentil $75 \%=4,60)$ y $0,25 \%$ (percentil $25 \%=0,14$; percentil $75 \%=0,42$ ). La disparidad de la prevalencia e IA crudas entre predios oscilaron entre $0 \%$ y $8,33 \%$ y $0 \%$ y $10,91 \%$ (figura 1 ).

\section{FACTORES DE RIESGO EN EL CONTEXTO DEL PREDIO LECHERO}

Considerando los DEL y la paridad conjuntamente y ajustando por el efecto del predio, para la prevalencia de $\mathrm{MC}$, solo los DEL ( $\leq 90$ días en leche vs $>90$ días en 
Cuadro 1. Prevalencia e Incidencia Acumulada de mastitis clínica estratificadas de acuerdo con la paridad y los días en leche ( $\mathrm{n}=2.535)$. Prevalence and Cumulative Incidence of clinical mastitis stratified by parity and days in milk $(n=2,535)$.

\begin{tabular}{lccc}
\hline Factores & Categorías & \% Prevalencia (IC 95\%) & \% Incidencia Acumulada (IC 95\%) \\
\hline Paridad & Vaca $(\mathrm{n}=1.592)$ & $3,14(2,25-4,02)$ & $5,31(4,16-6,47)$ \\
& Vaquillona $(\mathrm{n}=943)$ & $1,59(0,73-2,44)$ & $1,61(0,75-2,48)$ \\
DEL & $\leq 90$ días $(\mathrm{n}=650)$ & $4,15(2,54-5,76)$ & $4,49(2,78-6,20)$ \\
& $>90$ días $(\mathrm{n}=1.885)$ & $2,02(1,35-2,67)$ & $3,74(2,84-4,62)$ \\
\hline
\end{tabular}

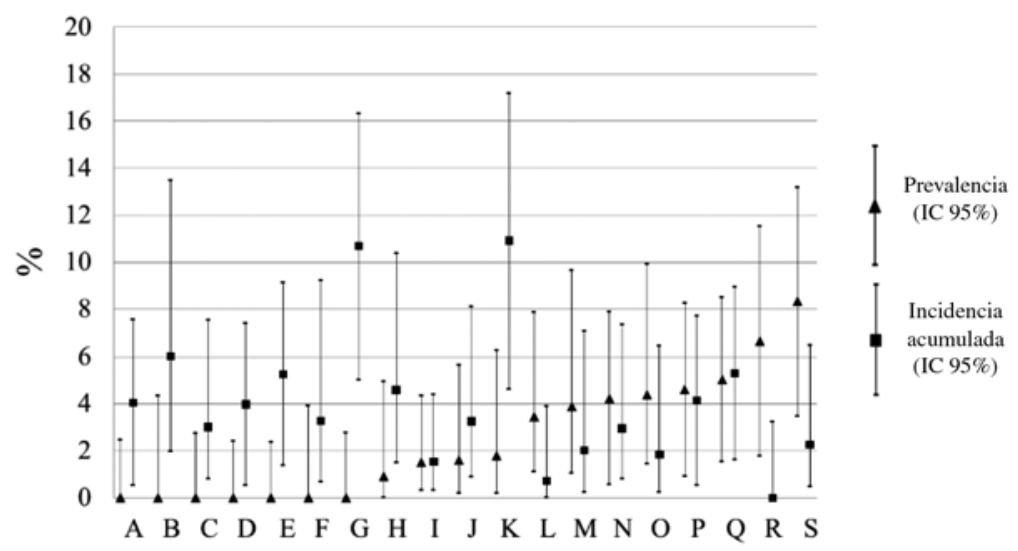

Predios lecheros

Figura 1. Prevalencia e incidencia acumulada de mastitis clínica (IC 95\%) estimadas para los establecimientos lecheros evaluados, Argentina (2011).

Clinical mastitis prevalence and cumulative incidence (CI 95\%) estimated for dairy herds from Argentina (2011).

leche) resultaron asociados con una OR de 1,69 (IC95\% $=1,01-2,85)$, mientras que para la IA la paridad fue la única variable asociada, experimentando las vacas una incidencia tres veces más alta respecto de vaquillonas $(\mathrm{OR}=3,36$; IC95\% = 1,84-6,11) (cuadro 2). De forma análoga, la TI fue casi 4 veces más elevada en vacas respecto de vaquillonas $(\mathrm{HR}=3,83 ; \mathrm{IC} 95 \%=2,05-7,15)$, sin evidenciarse diferencias significativas por los DEL. Según los CCI obtenidos, la variación en la prevalencia e IA de MC entre vacas podría ser atribuida en $29,30 \%$ y en $6,25 \%$ por el hecho de pertenecer a un predio en particular.

\section{ANÁLISIS ECONÓMICO}

Las pérdidas debidas al episodio de MC registrado (por leche descartada y tratamiento aplicado) para vaca y vaquillona fueron de US\$ 66,04 y US\$ 55,25.

En promedio las vacas presentaron el evento a los 154 DEL, mientras que en vaquillonas el mismo ocurrió a los 200 DEL. Las pérdidas para el resto de la lactancia por el caso de MC registrado, estimadas para estas situaciones genéricas, fueron de 321,55 y 237,25 litros para vaca y vaquillona que, traducidas a dólares, representaron un costo de US\$118,97 y US\$ 87,78.

Las pérdidas totales para un caso de MC fueron de US\$ 185,01 para vacas y de US\$143,03 para vaquillonas. Los costos promedios finales estimados para el total de vacas ( $\mathrm{n}=2.535)$ fueron de US\$ 4,50/VO (IC95\% = 3,38-5,61). Al examinar los costos a nivel de predio, los mismos oscilaron entre US\$ 0/VO y US\$ 15,54/VO, presentando la mitad de los predios un costo igual o superior a US\$2,43/VO.

\section{DISCUSIÓN}

Tanto en Argentina como en el resto de Latinoamérica son escasos los reportes acerca de valores de prevalencia e incidencia de MC a base de un número considerable de predios y, prácticamente, no se dispone de estudios con diseños probabilísticos. De aquellos trabajos publicados recientemente en nuestra región, Trujillo y col (2011) reportaron una prevalencia en vacas de 3,8\% (IC95\% = 1,4-6,2), Ramírez Vásquez y col (2011) de 1,7\% y Vissio y col (2015) de 3\% (IC95\%=2,3-3,7), valores similares a los registrados en este estudio al momento de la primera visita (prevalencia $=2,5 \%$; IC95\% $=1,9-3,1$ ). 
Cuadro 2. Factores asociados a las chances de casos prevalentes e incidentes de mastitis clínica.

Factors associated with the odds of being a prevalent or incident case of clinical mastitis.

\begin{tabular}{lccc}
\hline & $\begin{array}{c}\text { Casos } \\
\text { prevalentes }\end{array}$ & & $\begin{array}{c}\text { Casos } \\
\text { incidentes }\end{array}$ \\
\cline { 2 - 2 } & OR (IC 95\%)* & & OR (IC 95\%)* \\
\hline DEL** & $1,69(1,01-2,85)$ & & $1,12(0,70-1,78)$ \\
Paridad*** & $1,84(0,88-3,86)$ & & $3,36(1,84-6,11)$ \\
\hline
\end{tabular}

* Odds Ratio e Intervalo de Confianza (95\%) derivadas del modelo mixto con el rodeo como efecto aleatorio y ajustada por DEL y paridad.

** Días en leche ( $\leq 90$ días vs $>90$ días).

**** Vaca vs Vaquillona.

Debido a que un rodeo de VO puede considerarse básicamente como una población abierta, el cálculo de la TI es más apropiado que el de la IA (Thrusfield 2007). En nuestro caso, debido a que el período de seguimiento fue corto, ambas medidas pueden ser igualmente válidas (Thrusfield 2007). Por lo demás, los valores de incidencia demostraron una gran disparidad entre los predios evaluados $(\mathrm{IA}=0$ a $10,91 \%)$, lo que reflejaría la diversidad de situaciones potenciales que influencian la celeridad con la que la MC progresa en la población. Considerando la mediana de IA de rodeo $(3,6 \%)$ y de mantenerse con esta magnitud en el tiempo, se podría inferir que para la mitad de los predios una gran proporción de las vacas experimentarían al menos un caso al cabo de un año.

Es dificultoso cotejar los datos de incidencia estimados respecto de los reportados en estudios previos. Esto se debe, en parte, a la diversidad en cómo la incidencia ha sido calculada: mensual, trimestral, estacional (Shpigel y col 1998), lactacional (Wolfová y col 2006, Petrovski y col 2009), o si esta ha sido ajustada por otros factores (Pérez-Cabal y col 2008, Petrovski y col 2009, De Vliegher y col 2012). Además, discrepancias posibles entre la bibliografía pueden deberse a diferencias en la definición de caso (en relación con el curso de la enfermedad y la severidad de la misma), así como también en la detección del mismo, según quiénes fueron los encargados en hacer el diagnóstico (veterinarios, productores, tamberos, etc.) (Thrusfield 2007). Dichos elementos pueden provocar una subestimación o sobreestimación de la frecuencia de MC de cada predio (Martin y col 1987). Este último aspecto no puede ser descartado totalmente en nuestro trabajo, a pesar de que se hizo un esfuerzo en la sistematización de la definición de caso para minimizar el sesgo de detección.

Al considerar los DEL y la paridad de la vaca conjuntamente en el modelo multivariado, con el predio como efecto aleatorio, solo se observó un incremento significativo en las chances de casos incidentes en favor de las vacas, lo que es coincidente con previas investigaciones (Rajala-Schultz y col 1999, Wilson y col 2004, Breen y col 2009). En contraposición, algunos autores señalaron un mayor riesgo en vaquillonas, especialmente durante los primeros 14 días postparto (Nyman y col 2007, Olde Riekerink y col 2008, Steeneveld y col 2008). Estos hallazgos, en alguna medida, reflejarían además un efecto de la lactancia temprana sobre la incidencia de los casos clínicos, lo que ha sido también observado en vacas por Pérez-Cabal y col (2008), Breen y col (2009), Petrovski y col (2009), pero que no fue evidenciada en este estudio.

El grado de correlación del riesgo de $\mathrm{MC}$ de cada vaca dentro del rodeo es expresado por el coeficiente de correlación intraclase (Snijder y Bosker 2011). En este sentido, la proporción de varianza capturada por el conglomerado (rodeo) para casos prevalentes $(29,30 \%)$ fue superior al 15\% reportado por Vissio (2012), y sugiere que una porción significativa de la variación en la prevalencia de $\mathrm{MC}$ reside en aspectos vinculados al manejo de los predios. En contraposición, la mayor variabilidad en la ocurrencia de casos incidentes no se encuentra condicionada críticamente por el predio de pertenencia $(6,25 \%)$, lo que implica que el riesgo varía fundamentalmente por particularidades entre los individuos. La búsqueda de predictores individuales de riesgo de $\mathrm{MC}$ en vacas ha sido investigada extensamente por Steeneveld y col (2008), los que encontraron que antecedentes como la estación del año, los recuentos de células somáticas en leche en el mes anterior, la media geométrica de dichos recuentos en la lactancia previa y la historia de casos de MC, influyen significativamente en el riesgo de presentar un episodio clínico. Dichos factores no pueden ser descartados como influyentes en la variación individual del riesgo de casos incidentes en nuestro estudio.

Existen iniciativas en Argentina conducentes a valorar el impacto económico de la mastitis. Estimaciones previas de los costos deducidos a partir de datos de recuento celular somático en tanque fueron realizadas comparando predios que aplicaban buenas y malas prácticas de manejo (Richardet y col 2012). Por otra parte, Vissio y col (2015) en un estudio que involucró a 48 predios de Villa María, Córdoba, reportaron pérdidas por MC de $4 \mathrm{cc}$ de US\$/vaca/ día, cifras marcadamente inferiores a las estimadas aquí, debido a que el análisis solo incluyó costos de tratamiento y leche descartada de un día.

Las estimaciones presentadas en nuestro estudio describen de forma más completa el impacto económico de un caso, ya que contempla el retiro de leche completo y el efecto ulterior sobre la producción a lo largo de la lactancia. Aun así, durante el término de la lactación una vaca puede experimentar más de un evento de $\mathrm{MC}$ y un mayor nivel de pérdidas (Bar y col 2007), fenómeno que no fue evaluado a causa de los límites del período de monitoreo definido en el diseño del estudio. La investigación de dos o más repeticiones de MC implica una perspectiva de análisis por lactación, mientras que el propósito del presente estudio fue estimar más integralmente las consecuencias económicas a partir de un caso. 
Una de las falencias de esta investigación es que el diseño no fue probabilístico en lo que respecta a los predios, razón por la que los resultados no pueden ser extrapolados mecánicamente al sector lechero argentino. Además, los costos de tratamiento no fueron adaptados a cada predio debido a que no se colectaron datos de los protocolos empleados.

En un futuro sería de interés estudiar los efectos de la MC a nivel lactacional conjuntamente con aquellos vinculados a mediano plazo, como son el descarte prematuro o muerte de los animales bajo nuestras condiciones de producción. Los resultados obtenidos en esta investigación contribuyen a un mejor conocimiento del impacto económico de esta enfermedad, debido a que no existen estudios previos en Argentina que documenten pérdidas por episodios clínicos más allá del período de finalización del tratamiento.

La gran variación en la frecuencia e impacto de la MC refleja la importancia de esta manifestación de la enfermedad y permite inferir la existencia de una proporción considerable de pérdidas evitables.

\section{AGRADECIMIENTOS}

A los productores participantes y a los integrantes de la comisión de mastitis de AProCal por su activa colaboración.

\section{REFERENCIAS}

Bar D, YT Gröhn, G Bennet, RN González, JA Hertl, HF Schulte, LW Tauer, FL Welcome, YH Schukken.2007. Effect of repeated episodes of generic clinical mastitis on milk yield in dairy cows. J Dairy Sci 90, 4643-4653.

Barkema HW, YH Schukken, TJGM Lam, ML Beiboer, G Benedictus, A Brand. 1999. Management practices associated with the incidence rate of clinical mastitis. J Dairy Sci 82, 1643-1654.

Bradley A J. 2002. Bovine mastitis: An evolving disease. Vet J 164, 116-128.

Breen JE, MJ Green, AJ Bradley. 2009. Quarter and cow risk factors associated with the occurrence of clinical mastitis in dairy cows in the United Kingdom. J Dairy Sci 92, 2551-2561.

De Vliegher S, LK Fox, S Piepers, S McDougall, HW Barkema. 2012 Invited review: mastitis in dairy heifers: nature of the disease, potential impact, prevention, and control. J Dairy Sci 95, 1025-1040.

Halasa T, M Nielen, RBM Huirne, H Hogeveen. 2009. Stochastic bioeconomic model of bovine intramammary infection. Livest Sci 124, 295-305.

Hogeveen H, K Huijps, TJGM Lam. 2011. Economic aspects of mastitis: New developments. New Zeal Vet J 59, 16-23.

Le CT. 1997. Applied categorical analysis. John Wiley, New York, USA, Pp 239-278.

Martin SW, AH Meek, P Willeberg. 1987. Veterinary epidemiology: Principles and methods. Iowa State University Press, Ames, USA, Pp 1-343.

Nielsen C. 2009. Economic impact of mastitis in dairy cows. PhD Thesis, Faculty of Veterinary Medicine and Animal Science, Department of Animal Breeding and Genetics, Uppsala, Sweden, Pp 1-81.
Nyman AK, T Ekman, U Emanuelson, AH Gustafsson, K Holtenius, K Persson Waller, CH Sandgren. 2007. Risk factors associated with the incidence of veterinary-treated clinical mastitis in Swedish dairy herds with a high milk yield and a low prevalence of subclinical mastitis. Prev Vet Med 78, 142-160.

Olde Riekerink RGM, HW Barkema, H Stryhn. 2007. The effect of season on somatic cell count and the incidence of clinical mastitis. J Dairy Sci 90, 1704-1715.

Olde Riekerink RGM, HW Barkema, DF Kelton, DT Scholl. 2008. Incidence rate of clinical mastitis on Canadian dairy farms. J Dairy Sci 91, 1366-1377.

Pérez-Cabal MA, S Yaici, R Alenda. 2008. Clinical mastitis in Spanish dairy cows: incidence and costs. Span JAgric Res (España) 6, 615-622.

Petrovski KR, C Heuer, TJ Parkinson, NB Williamson. 2009. The incidence and aetiology of clinical bovine mastitis on 14 farms in Northland, New Zealand. New Zeal Vet J 57, 109-115.

Rajala-Schultz PJ, YT Gröhn, CE McCulloch, CL Guard. 1999. Effects of clinical mastitis on milk yield in dairy cows. J Dairy Sci 82, 1213-1220.

Ramírez Vásquez N, O Arroyave Henao, M Cerón-Muñoz, M Jaramillo, J Cerón, LG Palacio. 2011. Factores asociados a mastitis en vacas de la microcuenca lechera del altiplano norte de Antioquia, Colombia. Rev Med Vet 22, 31-42.

Richardet M, C Vissio, L Odierno, AJ Larriestra. 2012. Implicancias económicas de buenas prácticas dirigidas a promover bajos recuentos en tanque en productores de la cuenca lechera de Villa María. Resúmenes del $35^{\circ}$ Congreso Argentino de Producción Animal, Córdoba, Argentina.

Shpigel NY, M Winkler, G Ziv, A Saran. 1998. Clinical, bacteriological and epidemiological aspects of clinical mastitis in Israeli dairy herds. Prev Vet Med 35, 1-9.

Snijders TAB, RJ Bosker. 2011. Multilevel Analysis: An Introduction to Basic and Advanced Multilevel Modelling. Sage. $2^{\text {nd }}$ ed. London, UK.

Steeneveld W, H Hogeveen, HW Barkema, J van den Broek, RBM Huirne. 2008. The influence of cow factors on the incidence of clinical mastitis in dairy cows. J Dairy Sci 91, 1391-1402.

Tirante L, M Gavidia, C Vissio, L Odierno, AJ Larriestra. 2013. Effect of clinical mastitis on milk production. $52^{\text {nd }}$ National Mastitis Council Meeting, San Diego, California, USA.

Thrusfield M. 2007. Veterinary Epidemiology. $3^{\text {rd }}$ ed. Blackwell Science, Oxford, UK, Pp 1-593.

Trujillo CM, AF Gallego, N Ramírez, LG Palacio. 2011. Prevalence of mastitis in dairy herds in Eastern Antioquia. Revista Colombiana de Ciencias Pecuarias 24, 11-18.

Vissio C. 2012. Economic Impact of Mastitis in Dairy Cows. Tesis doctoral. Facultad de Agronomía y Veterinaria, Río Cuarto, Argentina, Pp 1-117.

Vissio C, DA Agüero, CG Raspanti, LM Odierno, AJ Larriestra. 2015. Pérdidas productivas y económicas diarias ocasionadas por la mastitis y erogaciones derivadas de su control en establecimientos lecheros de Córdoba, Argentina. Arch Med Vet 47, 7-14.

Wilson DJ, RN González, J Hertl, HF Schulte, GJ Bennett, YH Schukken, YT Gröhn. 2004. Effect of clinical mastitis on the lactation curve: A mixed model estimation using daily milk weights. J Dairy Sci 87, 2073-2084.

Wolfová M, M Štípková, J Wolf. 2006. Incidence and economics of clinical mastitis in five Holstein herds in the Czech Republic. Prev Vet Med 77, 48-64.

Zadoks RN, JL Fitzpatrick. 2009. Changing trends in mastitis. Irish Vet J 62, 59-70. 